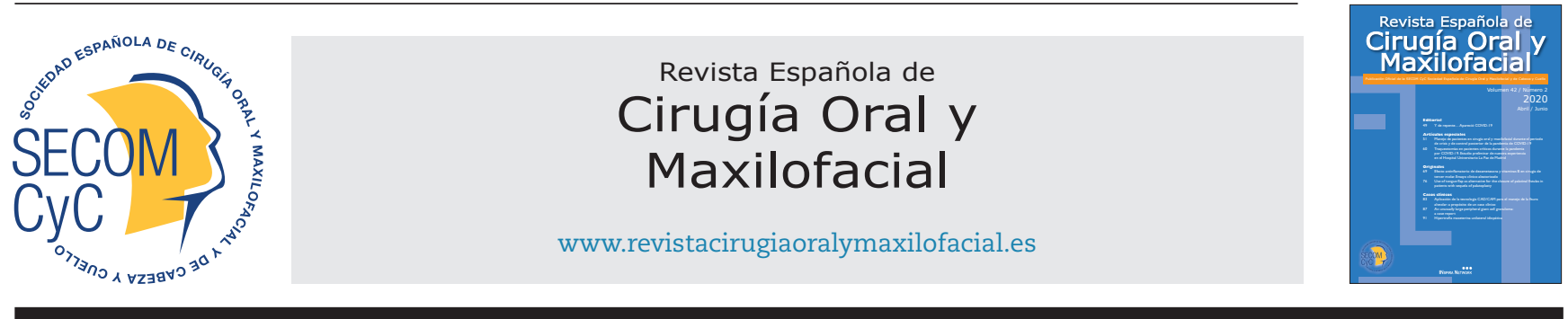

Caso clínico

\title{
Aplicación de la tecnología CAD/CAM para el manejo de la fisura alveolar: a propósito de un caso clínico
}

\author{
Adaia Valls-Ontañón, Laura Pozuelo-Arquimbau, Silvia Cuscó-Albors \\ y Antonio Marí-Roig
}

Hospital Universitario de Bellvitge, Hospitalet de Llobregat. Barcelona, España

\section{INFORMACIÓN DEL ARTÍCULO}

Historia del artículo:

Recibido el 28 de julio de 2019

Aceptado el 25 de octubre de 2019

\section{Palabras clave:}

Fisura alveolar, tecnología CAD/CAM, hemi-Le Fort I, osteotomía maxilar segmentada.

\section{R E S U M E N}

El paciente fisurado requiere un abordaje multidisciplinar e individualizado, siendo imprescindible adaptar la secuencia de tratamiento a cada paciente, según su situación clínica, sus necesidades y sus expectativas. Uno de los problemas más frecuentes del paciente fisurado es, entre otros, el colapso transversal del maxilar. La tecnología CAD/CAM nos permite la planificación virtual e impresión 3D, facilitando el abordaje y tratamiento integral del paciente fisurado en la actualidad.

El propósito de este artículo es mostrar el manejo de la fisura alveolar de un paciente adulto con fístula oronasal y maloclusión dental por colapso transversal del maxilar, en el cual se aplica la tecnología CAD/CAM para optimizar los tiempos quirúrgicos y de rehabilitación dental protésica. Discutir acerca de las posibilidades terapéuticas y los beneficios de la implementación de los avances en técnicas CAD/CAM.

\section{Application of CAD/CAM technology for alveolar cleft management: a case report}

\section{A B S T R A C T}

Cleft patient requires a multidisciplinary and individualized approach, being essential to adapt the treatment sequence to each patient, according to their clinical situation, their needs and their expectations. One of the most frequent problems of the cleft patient is, among others, the transverse collapse of the maxilla. CAD/CAM technology allows virtual planning and 3D printing that facilitates the comprehensive approach and treatment of the cleft patient today. The purpose of this article is to show the management of the alveolar cleft of an adult patient with oronasal fistula and dental malocclusion due to transverse maxillary collapse, in which CAD/CAM technology is applied to optimize surgical and prosthetic dental rehabilitation times. Discuss the therapeutic possibilities and benefits of implementing advances in CAD/CAM techniques.

\footnotetext{
*Autor para correspondencia:

Correo electrónico: Lauriarquim@gmail.com (Laura Pozuelo Arquimbau MD).
} 


\section{INTRODUCCIÓN}

El paciente fisurado requiere un abordaje multidisciplinar e individualizado, siendo imprescindible adaptar la secuencia de tratamiento a cada paciente, según su situación clínica, sus necesidades y sus expectativas.

Específicamente, centrándonos en el manejo de la fisura alveolar, la falta de hueso a dicho nivel suele asociarse a los siguientes problemas: fístula oronasal, maloclusión dental por colapso transversal del maxilar, ausencia del incisivo lateral y falta de erupción del canino, entre otros ${ }^{1}$.

Por otro lado, la implementación de la tecnología CAD/ CAM (Computer-Aided Design/Computer-Aided Manufacturing) en el ámbito quirúrgico nos permite diseñar virtualmente y luego imprimir en tres dimensiones (3D) todo tipo de férulas quirúrgicas y prótesis dentales, hechas totalmente a medida del paciente ${ }^{2}$.

El propósito de este artículo es mostrar el manejo de la fisura alveolar de un paciente adulto con fístula oronasal y maloclusión dental por colapso transversal del maxilar, en el cual se aplica la tecnología CAD/CAM para optimizar los tiempos quirúrgicos y de rehabilitación dental protésica.

\section{CASO CLÍNICO}

Varón de 45 años sin antecedentes de interés, salvo fisura labio-alveolar izquierda intervenida quirúrgicamente en la infancia mediante queiloplastia, sin reparación del hueso alveolar. Acudió a nuestra consulta buscando solución a su fisura alveolar productiva e insuficiencia respiratoria.

A la exploración física destacaba un perfil facial cóncavo con hipoplasia maxilar y laterorrinia. El examen intraoral evidenciaba una fístula con comunicación oro-nasal por ausencia de hueso alveolar a nivel de la fisura, confirmada mediante tomografía computarizada (Figura 1). A nivel dental presentaba colapso maxilar transversal y mordida cruzada izquierda, ausencia de incisivo central izquierdo rehabilitado con prótesis dentosoportada de 11 a 24 y edentulismo del resto del sector posterior superior izquierdo.

Como primera opción de tratamiento se propuso realizar una cirugía ortognática bimaxilar (Le Fort I en 2 piezas) con injerto de cresta ilíaca y rinoseptoplastia, pero el paciente rechazó el abordaje mandibular porque a nivel estético estaba satisfecho, y además quería acortar al máximo el tiempo postoperatorio por motivos laborales. Así pues, se optó por realizar un hemi-Le Fort I izquierdo (u osteotomía maxilar segmentada) para reposicionar la hemiarcada izquierda, un injerto de cresta iliaca para dar continuidad a la arcada superior y una rinoseptoplastia con motivos sobre todo funcionales.

La cirugía se planificó virtualmente con un software específico (Dolphin ${ }^{\circledR}$ 3D Orthognathic Surgery Planning Software Version 11.8) a partir de los archivos DICOM del TC facial y el .stl (stereolithographic file format) del escaneado intraoral digital (Lava Scan ST Scanner; 3M ESPE, Ann Arbor, MI, EE. UU.). La posición final de la hemiarcada fisurada se decidió tomando como referencia la dirección de oclusión de los pilares dentales superiores con los dientes de la arcada inferior. La férula quirúrgica se diseñó y fabricó mediante CAD/CAM de acuerdo con nuestro plan quirúrgico. Además, se le añadió una barra palatina de refuerzo para ayudar a evitar la recidiva transversal posterior inmediata. Por otro lado, se trasladó el archivo .stl de la arcada final al software 3Shape Dental System 2012 para diseñar y confeccionar mediante CAD/CAM una prótesis dental inmediata, la cual adaptaba a la perfección a los pilares dentales y a la nueva oclusión (Figura 2A).

La cirugía se llevó a cabo bajo anestesia general y según el plan previsto: instalación de tornillos de bloqueo intermaxilar,

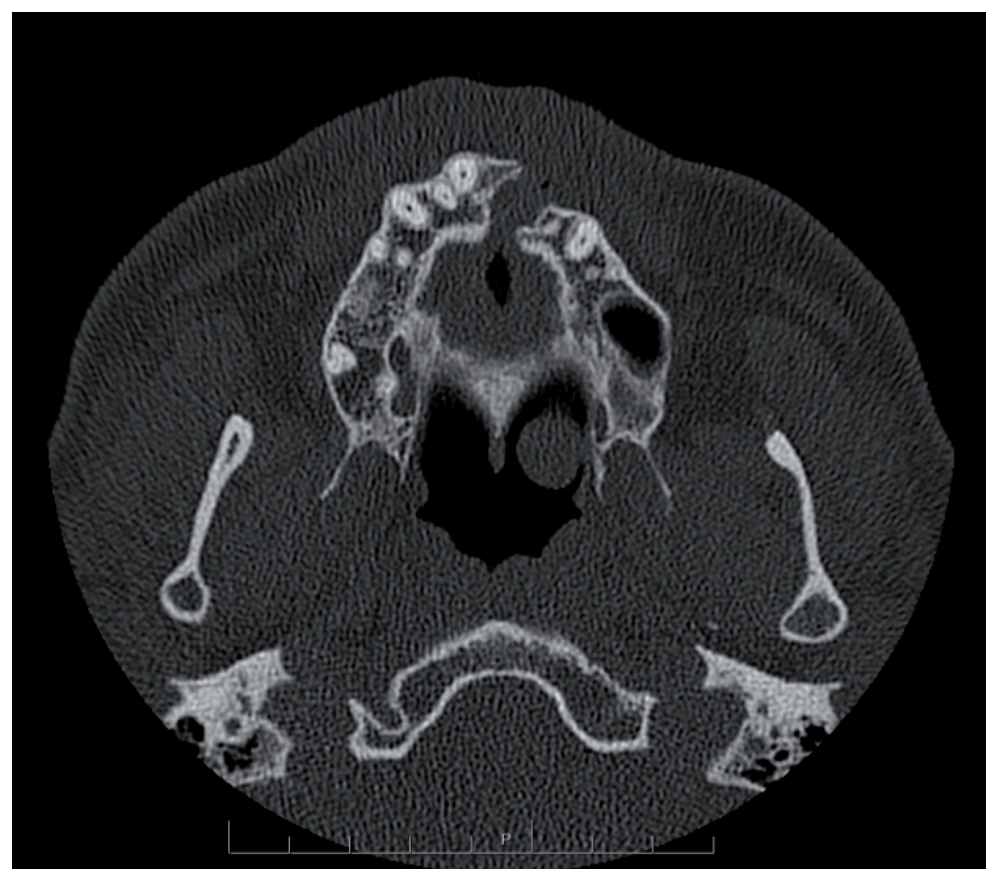

Figura 1. Imágenes axiales de tomografía computarizada preoperatorio. 

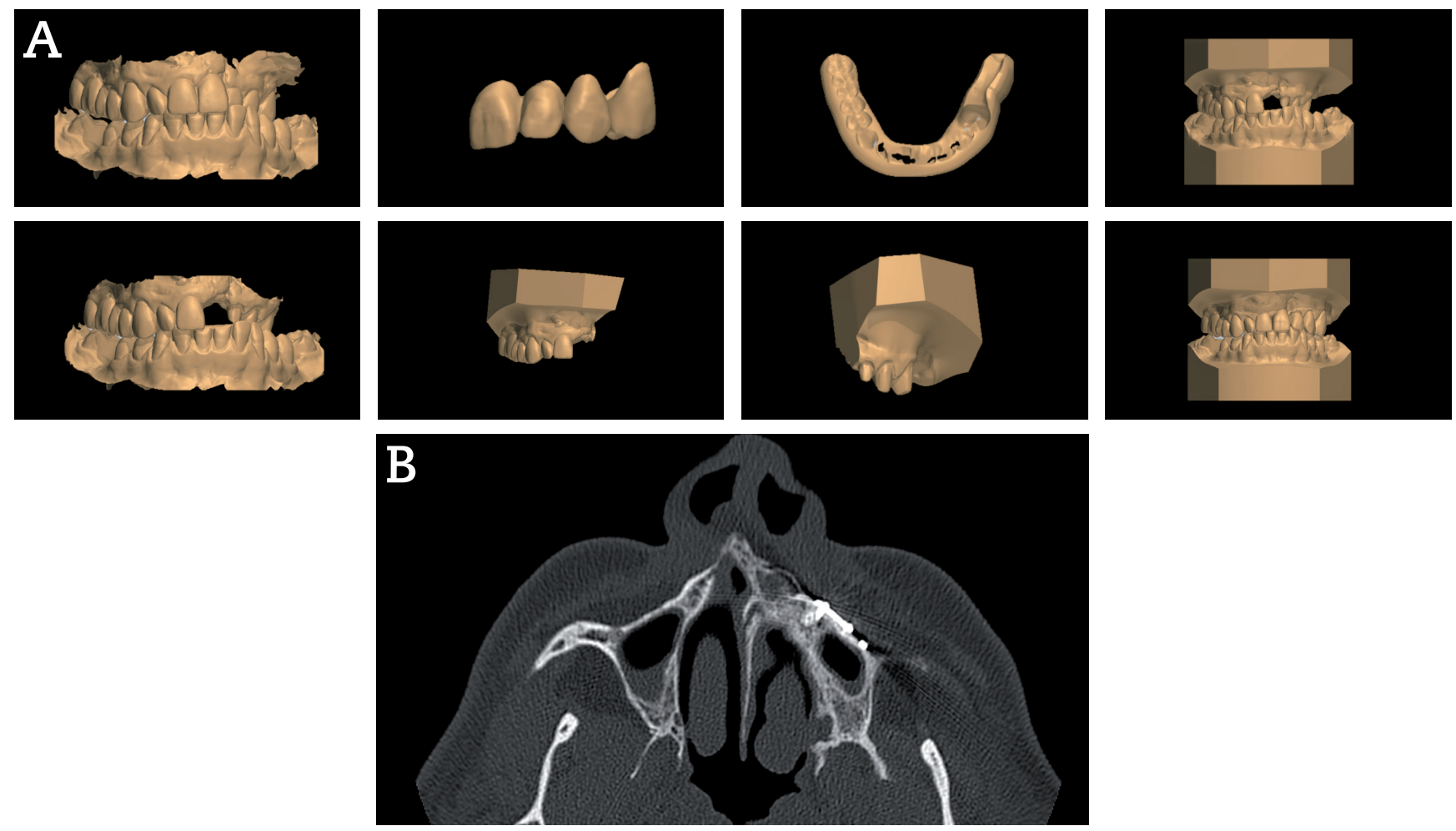

Figura 2. A. Planificación virtual 3D de la cirugía de reposición del maxilar fisurado, diseño CAD/CAM de prótesis dentosoportada inmediata. B. Imágenes axiales de tomografía computarizada postoperatorio al año postintervención.
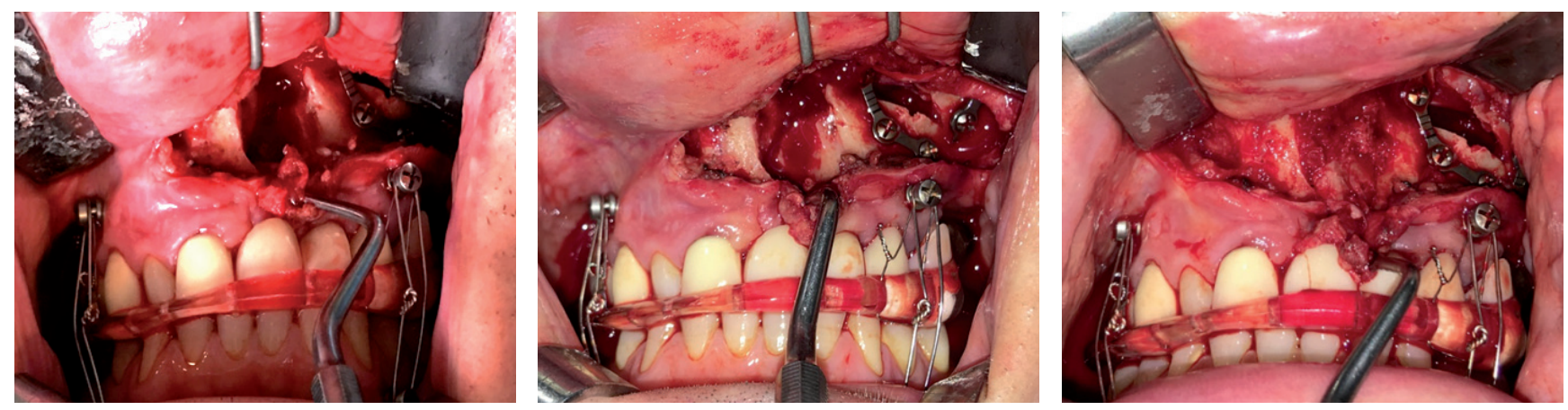

Figura 3. Fotografías intraoperatorias de la fisura alveolar, el Le Fort segmentado y el injerto de hueso.

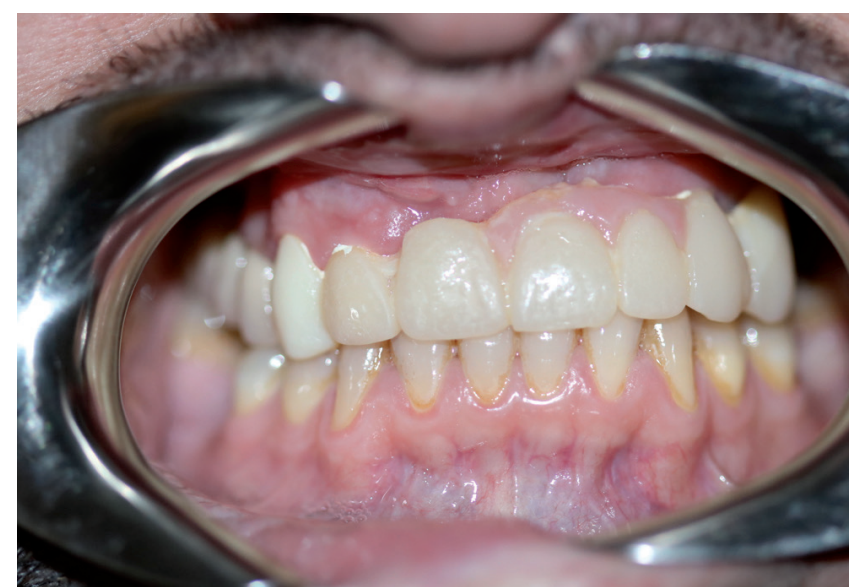

Figura 4. Registro fotográfico oclusal final. realización de osteotomía maxilar segmentada izquierda, colocación y fijación de prótesis dental y férula quirúrgica con bloqueo intermaxilar, reposición y osteosíntesis de hemiarcada izquierda con dos miniplacas y ocho tornillos monocorticales (KLS Martin, Jacksonville, FL, EE. UU.), obtención de injerto córtico-esponjoso de cresta ilíaca para el defecto óseo alveolar (Figura 3) y, finalmente, rinoseptoplastia abierta realizándose reducción de cornete inferior izquierdo, septoplastia transmaxilar y simetrización y remodelado de punta nasal mediante suturas e injerto de columnela sin necesidad de cambiar durante el procedimiento la intubación nasal ${ }^{3}$.

$\mathrm{Al}$ año de la intervención el paciente refiere satisfacción absoluta, manteniendo estabilidad oclusal con una prótesis metal-cerámica fija cementada, dentosoportada, ausencia de comunicación oronasal y resolución de la insuficiencia respiratoria. (Figura 2B y 4). 


\section{DISCUSIÓN}

Existen múltiples filosofías para el abordaje de las fisuras alveolares, referentes a la edad, el lugar de obtención del injerto o el uso de sustitutivos óseos y los tiempos de ortodoncia. Sin embargo, en la práctica clínica es imperativo confeccionar un tratamiento adaptado a cada paciente, como ocurre en el caso presentado. A continuación vamos a discutir los puntos clave del manejo del caso presentado (anteriormente detallado): osteotomía maxilar segmentada combinada con injerto óseo de cresta ilíaca y rehabilitación dental inmediata, con planificación quirúrgica virtual y tecnología CAD/CAM.

Por un lado, la realización de una osteotomía maxilar segmentada (hemi-Le Fort I) en pacientes con colapso transversal y sin discrepancia maxilomandibular sagital permite corregir la oclusión sin necesidad de ortodoncia, o bien acortando los tiempos de esta ${ }^{4}$. En el caso presentado, la magnitud de colapso transversal del maxilar fisurado impedía solventar la mordida cruzada mediante ortodoncia ni compensación protésica, por lo que se optó por llevar a cabo la reposición quirúrgica del segmento maxilar fisurado.

Por otro lado, el injerto óseo alveolar permite cerrar la fístula oronasal y restablecer la continuidad ósea del arco maxilar en el paciente adulto, mejorando la simetría nasal, el habla, el mantenimiento de la higiene bucal y la salud periodontal y, a su vez, mantener estable la arcada dento-alveolar ${ }^{5,6}$, evitando el colapso o recidiva transversal después de una cirugía ortognática o de una osteotomía maxilar segmentada7. Además, posibilita realizar un tratamiento de ortodoncia en condiciones y proporciona una estructura ósea con recubrimiento mucoso adecuado para una eventual colocación de implantes dentales osteointegrados ${ }^{8}$.

Llevar a cabo la osteotomía maxilar segmentada y el injerto óseo de forma concomitante es un procedimiento seguro y predecible ${ }^{9}$, pero hay que destacar que la mayor tasa de éxito del injerto alveolar se consigue cuando este se realiza antes de que el canino vaya a erupcionar ${ }^{10}$, ya que esto permite la erupción de la dentición permanente en el hueso injertado, a parte de limitar la alteración del crecimiento del maxilar superior fisurado ${ }^{5,6}$.

En cuanto al tipo de hueso, la evidencia científica corrobora que el injerto de hueso esponjoso se incorpora más eficazmente al alveolo que el hueso cortical en bloque ${ }^{6}$, pero el hecho de añadir hueso cortical por encima del injerto esponjoso incrementa la estabilidad de la arcada, a la vez que ayuda a compactar los chips de hueso esponjoso ${ }^{11}$.

Paralelamente, la confección de una prótesis dental inmediata customizada mediante tecnología CAD/CAM permite una rehabilitación dental inmediata, a la vez que incrementa la estabilidad transversal postoperatoria del maxilar segmentado $^{2}$. Además, en casos de edentulismo parcial como el presentado, la prótesis aumenta la estabilidad intraoperatoria permitiendo fijar la férula quirúrgica.
A nivel protésico, el uso de la tecnología CAD/CAM para la fabricación de prótesis fijas parciales tiene como ventajas: la aplicación de nuevos materiales, reducción del tiempo de edentulismo y de tratamiento, mejora de la relación coste-efectividad y su utilización como control de calidad ${ }^{12}$. En definitiva, los avances en planificación virtual y tecnología CAD/CAM, junto con la evolución de las técnicas quirúrgicas, optimizan el acto quirúrgico aumentando la precisión y predictibilidad de este, disminuyen el tiempo operatorio y la comorbilidad y, finalmente, acortan el tiempo de recuperación postoperatoria y facilitan la inserción social y laboral temprana del paciente.

\section{B I B L I O G R A F Í A}

1. Rosenstein SW. Early bone grafting of alveolar cleft deformities. J Oral Maxillofac Surg. 2003;61(9):1078-81. DOI: 10.1016/ s0278-2391(03)00321-5.

2. Benington PC, Khambay BS, Ayoub AF. An overview of threedimensional imaging in dentistry. Dent Update. 2010;37(8):4946. DOI: 10.12968/denu.2010.37.8.494.

3. Molins G, Valls A, Guijarro-Martínez R, Masiá J, de Nadal M, Hernández-Alfaro F. Simultaneous orthognathic surgery and rhinoplasty under nasotracheal intubation. J Clin Anesth. 2019;55:24-5. DOI: 10.1016/j.jclinane.2018.12.031.

4. Wilson AT, Wu RT, Sawh-Martinez R, Steinbacher DM. Segmental Maxillary Osteotomy to Close Wide Alveolar Clefts. J Oral Maxillofac Surg. 2019;77(4):850.e1-850.e5. DOI: 10.1016/j. joms.2018.11.011.

5. Seifeldin SA. Is alveolar cleft reconstruction still controversial? (Review of literature). Saudi Dent J. 2016;28(1):3-11. DOI: 10.1016/j.sdentj.2015.01.006.

6. Eppley BL, Sadove AM. Management of alveolar cleft bone grafting--state of the art. Cleft Palate Craniofac J. 2000;37(3):22933. DOI: 10.1597/1545-1569_2000_037_0229_moacbg_2.3.co_2.

7. Thongdee P, Samman N. Stability of maxillary surgical movement in unilateral cleft lip and palate with preceding alveolar bone grafting. Cleft Palate Craniofac J. 2005;42(6):664-74. DOI: 10.1597/04-042r.1.

8. Wang F, Wu Y, Zou D, Wang G, Kaigler D. Clinical outcomes of dental implant therapy in alveolar cleft patients: a systematic review. Int J Oral Maxillofac Implants 2014;29:1098-105.

9. Erbe M, Stoelinga PJ, Leenen RJ. Long-term results of segmental repositioning of the maxilla in cleft palate patients without previously grafted alveolo-palatal clefts. J Craniomaxillofac Surg. 1996;24(2):109-17. DOI: 10.1016/s1010-5182(96)80022-9.

10. Goudy S, Lott D, Burton R, Wheeler J, Canady J. Secondary alveolar bone grafting: outcomes, revisions, and new applications. Cleft Palate Craniofac J. 2009;46(6):610-2. DOI: 10.1597/08126.1.

11. Cho-Lee GY, García-Díez EM, Nunes RA, Martí-Pagès C, SieiraGil R, Rivera-Baró A. Review of secondary alveolar cleft repair. Ann Maxillofac Surg. 2013;3(1):46-50. DOI: 10.4103/2231 0746.110083.

12. Miyazaki T, Hotta Y, Kunii J, Kuriyama S, Tamaki Y. A review of dental CAD/CAM: current status and future perspectives from 20 years of experience. Dent Mater J. 2009;28(1):44-56. DOI: 10.4012/dmj.28.44. 